\title{
Propuestas Para Más Democracia del Conocimiento Académico en Comunicación
}

https://doi.org/10.21814/uminho.ed.43.10

\section{Eduardo Villanueva Mansilla}

Departamento Académico de Comunicaciones, Sección Comunicación, Pontificia Universidad Católica del Perú, Perú 



\section{Introducción}

La existencia de una tradición propia de estudio de la Comunicación, en América Latina, basada en el pensamiento crítico aplicado a la realidad social y económica de la región, es fácil de comprobar; esta tradición, nacida hace unos 40 años de la confluencia de perspectivas políticas y preocupaciones académicas, sigue presente en los temas y estilos de trabajo de los académicos latinoamericanos. Sin embargo, existen varios desafíos que en la actualidad debilitan el desarrollo y actualización de esta tradición.

Considerando específicamente la cuestión de la difusión del conocimiento creado por esta tradición académica, es necesario ponderar las dificultades específicas que surgen de las varias vertientes de producción. Mucho se hace, poco circula; sistemas nacionales altamente formalizados como el brasileño conviven con realidades de pocos recursos y baja circulación interna, ya no regional, que nos lleva a desconocer lo avanzado y en muchos casos a duplicar debates que no siempre requieren seguir siendo discutidos. Los profesionales de la comunicación aportan conocimiento directo, empírico, de la realidad comunicativa de nuestros pueblos; los académicos intentan sintetizar, recurriendo en muchos casos al ensayo antes que los estudios basados en datos. Tendencias globales temáticas y metodológicas atraviesan el debate específico y alteran la dinámica. Al mismo tiempo, continuidades concretas permiten reconocer lo que no ha cambiado a pesar de la transformación: la demanda por un sistema sociotécnico que permita sostener la creación de conocimiento de manera sostenible y útil a la sociedad (Borgman, 2007). Estas premisas son igualmente relevantes para América Latina, con tonalidades específicas que se discutirán a lo largo de este artículo.

La dimensión lingüística no es trivial. No solo tenemos la barrera objetiva entre el Español y el Portugués, que si bien son cercanos tienen suficientes diferencias para requerir competencias idiomáticas concretas. El idioma de circulación de la producción científica a nivel global es el Inglés, y producir en esa lengua, implica alejarse de muchos potenciales lectores de la región, no solo por el idioma mismo, sino porque se trata de un circuito de circulación completamente distinto, muchas veces, difícil de acceder para los académicos de la región. Al mismo tiempo, la demanda por internacionalización exige reconocer al Inglés como una fuente de documentación crítica y como un requerimiento de validación global del trabajo que se hace a nivel local.

Otro aspecto es la cuestión conceptual/terminológica. No todo lo que decimos se entiende igual en todas partes, y a veces la explicación singular de por qué llamamos algo como lo llamamos es tan particular que termina por complicar el diálogo. Discutir la terminología implica entender las singulares historias detrás 
de la producción académica, y por ello, es necesario incorporarlo en cualquier elaboración sobre el conocimiento registrado y difundido en la región.

Aunque no es posible proponer un estado de la cuestión respecto a estos asuntos - puesto que falta información y antes que eso, investigación sobre las condiciones de circulación de la investigación latinoamericana en Comunicación - es posible proponer algunos temas críticos y a partir de ellos, plantear posibles líneas de trabajo para el futuro, trabajo que puede estar enmarcado en organizaciones como la Confederación Iberoamericana de Asociaciones Científicas y Académicas de Comunicación (Confibercom), en alianza con asociaciones nacionales y con los organismos nacionales dedicados a estos temas.

\section{Publish or perish}

La demanda por publicación se ha agudizado en los últimos años, creándose al menos tres conflictos importantes.

\section{Core journals y el espacio de publicación relevante}

El dilema del impacto visto como citas, o la incidencia local (académica o política) sigue estando al centro de la conversación académica. La percepción de una competencia cada vez mayor por prestigio y estudiantes basada en la posición en los rankings requiere que las universidades promuevan la publicación de artículos en revistas de alta calificación, o core journals, como se les llama en Inglés. Estas revistas suelen estar publicadas por editoriales comerciales y requieren que las contribuciones se hagan en Inglés.

Es discutible que publicar en esas revistas sirva para algo más que ser reconocido entre pares, en el mejor de los casos. Se trata de productos editoriales caros, que presentan dos problemas completamente distintos: exigen escribir un discurso denso y complejo que está alejado de las preocupaciones profesionales o políticas de cada realidad nacional; al mismo tiempo, este discurso requiere métodos y objetivos distantes de lo que tiene impacto directo en la formación profesional en cada contexto. Esto aleja a los académicos que publican en revistas con altos factores de impacto tanto de la formación profesional como de la discusión de políticas públicas. El resultado es que nos conectamos con elites académicas globales, pero perdemos la relación con el entorno inmediato.

Los core journals presentan otro inconveniente: el grado de esfuerzo que requieren para lograr ser aceptados suele obligar a desbrozar la especificidad local en favor de aproximaciones de interés genéricamente global, las que terminan siendo vistas como "ciencia", sin que por ello lo sea en el sentido de las ciencias más tradicionales, 
pero reforzando la pretensión de una mirada academicista de la realidad. Es parte de un juego político que demanda integrar a la academia regional a las prácticas globales, pero que no deja mucho margen que esta incorporación sea bajo condiciones medianamente igualitarias.

Pero es cierto que al menos en el campo de la Comunicación, América Latina se ha refugiado en producciones más ensayísticas, sin intención de replicabilidad o de relevancia más allá del entorno inmediato. Seguimos considerando lo latinoamericano como algo viable, pero no logramos crear un discurso sólido y consistente de nivel regional, conformándonos con coincidencias y similitudes; renunciamos a la observación sistemática en aras de la calidad expresiva.

Lograr equilibrio requiere un debate institucional fuerte: ¿a quién nos debemos? Y más importante aún, ¿cómo podemos ser realmente relevantes para aquellos a quienes nos debemos sin perder la capacidad de dialogar con el mundo en su conjunto? ¿Cúal es el canal para lograrlo? Los core journals son útiles, pero lejanos, y nuestras publicaciones son más de una vez demasiado inmediatas: se trata de reflejos de dos vectores de abordaje del trabajo académico, que en una versión simplificada implica la absoluta sumisión a modelos cientifistas, y, por el otro, la completa obediencia al debate cotidiano. Defender uno u otro a ultranza es absurdo, pero algún equilibrio es necesario proponer.

\section{Acceso abierto}

América Latina ha sido considerada como la región más adelantada en la adopción del acceso abierto a sus revistas científicas y académicas, gracias a iniciativas como SciElO, Redalyc, el portal de portales Latindex y la Red Federada de Repositorios Institucionales de Publicaciones Científicas (Fischman \& Alperin, 2015). Al mismo tiempo, tenemos muchos desafíos respecto a calidad, difusión y utilización de todos estos trabajos.

El mercado de la publicación académica comercial, en manos de conglomerados globales orientados a la ganancia antes que, en cualquier otra cosa, ha creado costos altos sin necesariamente satisfacer las necesidades de difusión de los autores. Apoyados en este modelo, las autoridades universitarias y educativas nacionales fuerzan a la publicación en revistas comerciales, caras y de difícil acceso, sin fomentar mecanismos alternativos de control de la calidad del trabajo académico.

Como contrapartida, parte de la comunidad académica internacional está respondiendo con el movimiento del acceso abierto, que busca liberar los contenidos y publicarlos a través de espacios definidos como libres, los que respetan a los autores al dejarles en sus manos el control sobre el uso de la publicación, y al público al 
permitirle usar los contenidos de manera libre, difundiendo con mucha más amplitud el producto de su trabajo.

Promover el acceso abierto consiste no solo en publicar en revistas abiertas o en editar publicaciones abiertas, sino en participar del debate y articular a la comunidad académica como actor en el desarrollo del movimiento. Organizaciones como la Asociación Latinoamericana de Investigadores de la Comunicación (ALAIC) cuentan con revistas abiertas (el Journal of Latin American Communication Research ${ }^{1}$ ) y universidades de la región han desarrollado, mediante el uso de plataformas libres como Open Journal Systems (OJS)², publicaciones en este espíritu. Es además importante considerar que OJS es parte de los esfuerzos del Public Knowledge Project, de la Universidad Simon Fraser, de Vancouver, Canadá ${ }^{3}$, que incluye plataformas para el descubrimiento de contenido ${ }^{4}$, para la publicación de monografías ${ }^{5}$ y para el manejo de conferencias ${ }^{6}$.

A estos servicios se les complementa con el directorio de publicaciones de acceso abierto (Directory of Open Access Journals [DOAJ]), que tiene estándares específicos para garantizar la calidad del acceso ofrecido, lo que hace que cada revista deba solicitar una evaluación previa para ser incorporados ${ }^{7}$. La seguridad de la información publicada, que requiere un repositorio Public Knowledge Project adecuado para la revista de manera de garantizar la integridad y continuidad del servicio, se complementa con LOCKSS (Lots Of Copies Keep Stuff Safe), manejado en la Universidad de Stanford, que permite que las bibliotecas participantes hagan copia local de los contenidos de una revista de manera que, en caso de problemas significativos con el repositorio, la integridad de la colección no se pierda ${ }^{8}$.

Pero falta valorar lo que esto significa, y sobre todo, convertir el acceso abierto en el nuevo sentido común de la práctica académica. Se trata de buscar que todas las publicaciones abiertas sean conocidas, que circulen ampliamente, pero también que los comités de evaluación valoren tanto a las revistas abiertas como a las comerciales, o que cuando se decida habilitar una nueva publicación siempre se diseñe considerando versiones digitales de libre disponibilidad.

\footnotetext{
${ }^{1}$ Ver www.alaic.net/journal/index.php/jlacr

2 Ver http://pkp.sfu.ca/?q=ojs

${ }^{3}$ Información retirada de http://pkp.sfu.ca

${ }^{4}$ Ver http://pkp.sfu.ca/harvester

${ }^{5}$ Ver http://pkp.sfu.ca/omp

${ }^{6}$ Ver https://pkp.sfu.ca/ocs/

${ }^{7}$ Ver https://doaj.org/

${ }^{8}$ Ver https://www.lockss.org/
} 
Además, resulta urgente difundir de manera agresiva estos medios de publicación, creando espacios de difusión de su existencia y que sirvan además de metasitios, que recojan las varias opciones existentes. Las facilidades tecnológicas para lograr esto son cada vez más asequibles, estando muchas veces en manos de las bibliotecas universitarias en las que cotidianamente buscamos nuevos contenidos.

Una alianza con la comunidad bibliotecaria, más una promoción de la discusión intelectual sobre la necesidad de nuevas políticas a nivel nacional, institucional y también individual, permitiría fomentar el acceso abierto más allá del movimiento hacia el sentido común, rompiendo además la lenta marcha hacia la estandarización en el estilo burocrático anglosajón que las reformas globalizantes de la actividad académica parecen hacer inevitable. Un reclamo por el openness es pues un alegato por la libertad académica y la integración de esfuerzos hacia un entendimiento cada vez más poderoso de nuestras realidades.

Como se ha mencionado, la sumisión a los rankings de índices de citas es un problema significativo. El estímulo fundamental para la publicación académica sigue siendo la carrera docente, que considera estos rankings como el indicador central. La abundancia de opciones no quiere decir que el modelo mismo sea divergente: mientras más citas tiene un artículo, más valioso el citado; mientras más artículos con muchas citas tiene una revista, su valor aumenta. Publicar en las revistas más "populares" se convierte en un destino, no en un camino. Las revistas abiertas no logran todavía convertirse en alternativas significativas, en especial, en ciertos campos como la Comunicación.

\section{Revistas depredadoras}

En el escenario contemporáneo, la aparición de revistas depredadoras es una cuestión que debe al menos diseminarse. Definimos una revista depredadora como aquella que cobra por la publicación de un artículo pero que no realiza ninguna de las funciones editoriales tradicionales de una revista académica, entre ellas, el arbitraje por pares, y que ofrece acceso abierto, pero sin participar de las herramientas habituales, como el directorio de revistas de acceso abierto (DOAJ) o servicios como el mencionado LOCKSS. Si bien existen muchos servicios que cobran por la publicación como un mecanismo de garantizar el acceso abierto - muchos de los cuales son provistos por editoriales comerciales - una revista depredadora simplemente publica sin control editorial alguno, cobrando por permitirle a cualquier decir que ha publicado.

Hay muchos criterios para definir una revista depredadora: la Asociación de Bibliotecarios de los EEUU ha definido 15 (Berger, 2017), donde uno de los más saltantes es la excesiva amplitud temática que se supone cubre: por ejemplo, un 
típico mensaje de correo enviado a la carpeta de spam proviene de una revista con el inverosímil título de International Journal of Latest Research in Engineering and Management, que además indica que cubre "todas las áreas de Ingeniería, Gestión Empresarial, Humanidades y muchas más”. Algunas de estas revistas son capaces de aceptar literalmente cualquier cosa, incluso, si solo consiste en una oración insultante repetida a lo largo del texto (Safi, 2014).

Si bien el criterio para separar las revistas depredadoras de las simplemente malas suele ser la intención de estafar al autor, ofreciendo datos falsos y similares, lo cierto es que un autor o supervisor desprevenido puede caer con facilidad; o un autor que quiere lograr acumular publicaciones simplemente puede optar por publicar sin criterio alguno. Parte de los excesos propios de la tendencia a publish or perish que comienza a volverse común en nuestra región, las revistas depredadoras son un peligro para la integridad académica que debe combatirse, y que requiere esfuerzos profesionales para lograr que no se difundan y que los autores, autoridades académicas y lectores no sean víctimas de sus prácticas.

\section{Nuevos medios y nuevos contenidos}

Precisamente en este espacio surge la necesidad de reconocer que la producción intelectual en Comunicación no se agota en publicaciones convencionales. Por un lado, las manifestaciones de la Comunicación, esos productos que constantemente llaman a la reflexión y renovación de las perspectivas intelectuales, solían estar fuera del alcance de quienes no podían viajar o que contaban con recursos para adquirir copias de distinto origen. Esta transformación ocurre en todos los campos de la investigación académica y se refleja en alternativas novedosas que no siempre resultan fáciles de apreciar y de compartir (Meyer \& Schroder, 2013).

La abundancia de contenidos y de nuevos formatos ahora disponible gracias a la internet ha cambiado la situación, pero muchas veces no encontramos cómo insuflar nuestra práctica académica de esos contenidos novedosos que reflejan además la creatividad de los latinoamericanos, más allá de las limitaciones impuestas por los sistemas comerciales de radiodifusión. Tenemos la oportunidad de atestiguar el cada vez mayor número de productos audiovisuales, en diversidad de medios y formatos, pero al mismo tiempo la preservación, para no decir la organización, de dicho material no está garantizada.

Temas emergentes, desde las nuevas narrativas que surgen en juegos de video, en video para la web, en formas de comunicación reiterativa como los memes; hasta las recreaciones y reformulaciones de tradiciones culturales latinoamericanas en la música, el video y la narrativa escrita, están inscritas en la internet gracias a la facilidad de acceso y de copiado que ahora se ha vuelto la norma. Promover el 
intercambio de estos imaginarios, facilitar el acceso a estas expresiones de creatividad, puede servir para descubrir con amplitud la diversidad en la similitud, la manera como los consumidores, los empresarios emergentes y también los académicos hacen el esfuerzo de aprovechar al máximo las capacidades expresivas de los medios digitales para ampliar el horizonte comunicativo de sus comunidades. Es posible argumentar que un nuevo campo, los llamados Estudios de Internet, comienza a alcanzar autonomía epistemológica a pesar de su clara deuda con los estudios de Comunicación (Dutton, 2013).

También los académicos crean en los nuevos medios: desde trabajos formativos en las universidades hasta nuevas maneras de explicar ideas o difundir conocimiento, los profesores e investigadores poco a poco comienzan a explorar esas posibilidades y no se limitan al trabajo habitual. Es indispensable promover la diseminación y el acceso a estas nuevas formas, lo que, en muchos casos, no requiere más que coordinar entusiasmos locales que se expresan en índices hechos por entusiastas en foros en donde se discute lo nuevo y lo novedoso.

Esto implica desarrollar capacidades para recoger, organizar y hacer fácil la recuperación de contenidos tanto empíricos como materiales de difusión y discusión académica que van más allá del formato escrito tradicional. No hay una solución real aun a la pérdida de lo que se hace en este ámbito, y es un asunto que debería preocuparnos: ¿cuantos de nosotros desarrollamos proyectos que dependían de formatos que han, para todo efecto práctico, desaparecido ya? El material en CD o DVD ha desaparecido salvo para la música el primero (mínimamente) y los videos el segundo (algo más común) y los formatos de software contenidos en su interior, difíciles de usar; videos que hace 10 años se veían bien en una computadora ahora son poco atractivos; los juegos de video se pierden casi por completo una vez que la plataforma en que fueron desarrollados se vuelve comercialmente obsoleta.

La producción audiovisual es ahora pensada para el streaming y la realidad es que las copias duras cada vez son menos populares, a nivel general. Esto crea problemas para la preservación de materiales y la continuidad de colecciones, pues los formatos tradicionales desaparecen, al quedar sin equipamiento para reproducirlo, ni permisos para hacer copias. En tiempos en que parecería natural esperar más acceso a la enorme producción cultural de la región, es más difícil por la abundancia de formatos, la complejidad de montar repositorios, y la dependencia de servicios como YouTube o similares, que ofrecen opciones con la complicación de ceder control sobre la explotación comercial o simplemente, facilitan la piratería. Salvo casos como la colección de preservación cultural que realiza la Biblioteca del Congreso de los EEUU, o las grandes filmotecas del mundo, el riesgo es la lenta desaparición de una diversidad mayúscula de producción audiovisual. No es que haya un sistema de preservación que garantice la supervivencia de cualquier y todo material. Salvo los 
libros bajo depósito legal, no es raro que se pierdan materiales en papel, el formato más seguro y estable que conocemos; la producción de películas en el mundo entero ha sufrido de desastres, descuidos o simple falta de valoración, con lo que mucho de lo que existió, ya se perdió. Los formatos obsoletos ya mencionados no solo desaparecen, son destruidos por razones comerciales. Un disquete puede guardar información valiosa, pero sin una máquina que lo lee y medidas básicas de preservación, da lo mismo que si el contenido hubiera sido borrado de la faz de la tierra.

Sin soluciones obvias, al menos ser conscientes de la fragilidad de los materiales, tanto de difusión como académico, es una obligación profesional. Nuevamente el dialogo con la comunidad bibliotecaria, que está constantemente desarrollando alternativas a la perdida de documentos, es necesaria. Similarmente, la necesidad de desarrollar políticas públicas que reconozcan la importancia de repositorios nacionales y regionales fuera de la lógica comercial es un tema que se debería al menos discutir. Proyectos de enorme valor como "The Internet Archive" ${ }^{9}$ deberían ser promovidos y discutidos ampliamente buscando alianzas para recoger lo que se produce en la región: no es una solución perfecta pero ayudaría mucho a acceder a lo existente, pero sobre todo a no perder lo que puede quedar obsoleto técnicamente.

\section{Lenguas y términos}

Un problema concreto es el idioma en el que escribimos, y otro la terminología que usamos. Si el lenguaje global de la comunicación académica es el Inglés, nuestra región habla Español y Portugués, y como es sabido, en contextos académicos o "cultos", las barreras para leer ambos idiomas son menores, aunque el intercambio verbal sea otra historia. Pero eso no significa que no exista una demanda cada vez mayor para escribir y comunicarse en Inglés.

Esto plantea tanto el asunto de cómo escribir y de por qué escribir en un idioma que aleja del grueso de estudiantes y de muchos colegas lo que se produce. Aparte de los inconvenientes asociados a la disponibilidad de los textos, la barrera idiomática complica la difusión del trabajo y hace más difícil que se pueda conocer lo que se hace en la región; no siempre es posible traducir lo que se publica en Inglés.

Pero si no se escribe en Inglés, la participación en eventos internacionales también se ve perjudicada. Nuestra región no ofrece la variedad y amplitud temática que existe en eventos globales, incluso sin considerar la rigidez metodológica que a veces hace inaccesible la investigación realizada en otras regiones. Al mismo tiempo, la relativa abundancia de ensayos y la poca vocación por replicabilidad en la investigación hace que muchas conferencias regionales presente pocas oportunidades de intercambio real.

\footnotetext{
${ }^{9}$ Ver www.archive.org
} 
También existe el problema de la terminología que se usa en la región, que no es trivial. Una rápida revisión de la literatura en Inglés permite constatar que términos que se siguen usando en América Latina, como NTIC (nuevas tecnologías de información y comunicación), han desaparecido de los estudios de Comunicación, reemplazados por medios digitales, entre otros; ¿por qué continuamos usando un término que pudo tener sentido hace 30 años, pero que ha sido abandonado por impreciso?

Similarmente, el uso de "redes sociales digitales" para referirnos a servicios como Facebook es más un tributo a la popularidad general del término que a la discusión académica global, donde se usa "medios sociales" hace más de una década, separando un concepto comunicacional como este del concepto sociológico de las redes sociales, lo que permite mayor precisión. Curiosamente, se hace más común hablar de social media, lo que es innecesario pues la traducción al Español es simple y sin complicaciones lexicográficas. Además, se pierde la potencia de la reflexión sobre la dimensión social de la Comunicación que involucra el uso del término "medio social” (Fuchs, 2014).

Resulta pues importante debatir qué nos hace divergir de las prácticas terminológicas de la academia global y cuál sería la justificación para hacerlo. Sin duda fenómenos específicos a nuestra realidad necesitan terminología propia; pero realmente ¿mantener cierta singularidad lingüística ayuda al desarrollo académico o simplemente complica el dialogo y nos impide renovar la conversación?

\section{Intercambio directo entre académicos}

Precisamente gracias a la tecnología digital, es posible proponerse el intercambio académico directo como objetivo. No se trata tan solo de las facilidades que herramientas como el correo electrónico, sino la posibilidad de integrar esfuerzos de muchos especialistas que pueden ya estar intercambiando, mediante foros o listas de correo, o quizá usando herramientas más sofisticadas como Mendeley ${ }^{10}$ o Zotero ${ }^{11}$, pero visibilizando este intercambio y valorándolo como la ruta a seguir.

Romper las limitaciones entre países debería ser una tarea para las asociaciones de investigadores y académicos, las cuales no deberían permitir que las prácticas hasta ahora sólidas e interesantes, pero como quedan en espacios privados, no sean aprovechadas por toda la comunidad académica.

No se trata de forzar que aquello que es privado sea público, pero sí de difundir las posibilidades. Crear espacios de discusión que difundan los mecanismos ya

\footnotetext{
${ }^{10}$ Ver www.mendeley.com

${ }^{11}$ Ver www.zotero.org
} 
existentes de intercambio entre académicos, pero que también expliquen cómo y qué tan fácil o difícil puede ser crearlos usando determinadas herramientas, puede servir para salir del localismo y también de la dictadura de los espacios coordinados por organismos oficiales, nacionales. Promover el control por parte de los mismos académicos de su producción requiere que también sea posible coordinar las acciones de intercambio básico que permiten luego ser creativo y productivo.

Estas tres líneas son pues en realidad una sola: aprovechar al máximo las oportunidades digitales para diseminar más y mejor tanto los insumos (la creatividad comunicativa en la región), los argumentos (a través de intercambios entre académicos) y la producción final, en la forma de publicaciones abiertas. Las bibliotecas académicas, muchas veces innovadoras en lo digital mucho más de lo que conocemos, pero desconocidas por académicos acostumbrados a una forma tradicional de trabajo, pueden ser aliadas importantes; los movimientos internacionales para la promoción de la openness también buscan diseminar sus ideas, sus propuestas de política y sus herramientas.

No se trata de ignorar las tradiciones y las formas ya consagradas de trabajo académico: se trata de innovar y facilitar al mismo tiempo que se promueve lentamente el cambio hacia un modelo más democrático e inclusivo de trabajo académico. Para eso, es necesario liderazgo, y es posible lograrlo sin necesidad de embarcarse en proyectos complejos, al menos no al inicio del proceso.

\section{Propuestas}

Confibercom, como espacio de encuentro de las comunidades académicas latinoamericana e ibérica, tiene la oportunidad de facilitar no solo el intercambio entre especialistas sino el cambio de prácticas basadas en tradiciones nacionales. Es sin duda respetable el trabajo que cada país logra desarrollar, pero la gran ventaja del espacio lingüístico que compartimos, la facilidad de intercambio de ideas, no es aprovechada al máximo a pesar de la existencia de oportunidades cortesía de la tecnología pero sobre todo, de la coincidencia de temáticas y de métodos de trabajo. Además, mediante este tipo de intercambio sería más fácil enfrentar la necesidad de fortalecer metodológicamente el campo y actualizar la aproximación conceptual a algunos temas que son estudiados con marcos conceptuales mucho más desarrollados en países en el ámbito cultural y universitario anglosajón.

La promoción del acceso abierto (AA) requiere no solo la creación de espacios tales como revistas, sino la afirmación política del AA como práctica estándar de la comunidad académica de la Comunicación. Sin duda poner todas las publicaciones que haga Confibercom en un espacio abierto es esencial, pero lograr que la práctica sea conocida por todos los académicos y sobre todo por todos los administradores 
universitarios para que se afirme la decisión política de publicar abiertamente es algo que solo se puede lograr desde una mirada integradora y sistemática, precisamente aquella que una instancia como Confibercom puede ser.

Por ello, Confibercom puede difundir los principios del AA; participar en foros de promoción de AA; involucrarse en los debates internacionales sobre el mismo; y promover la discusión académica desde la reflexión comunicativa, de manera que se integren saberes académicos propios de los estudios que realizamos con la actividad académica de difusión del conocimiento. Además, promover la difusión de contenidos bajo principios de AA mediante un espacio de difusión en su sitio web, o a través de la identificación de espacios con esas características ya existentes para que la comunidad académica tenga la oportunidad de conocer mejor lo que está ya disponible para compartir entre todos, y también se promueva las ventajas del acceso abierto.

Precisamente, conectar a los académicos requiere ofrecer también contenidos que se puedan usar como base para la reflexión. Existen iniciativas dispersas, desde el tradicional concurso de documentales que la Federación Latinoamericana de Facultades de Comunicación Social (Felafacs) realiza en cada encuentro hasta concursos locales; pero lo que podría potenciar aún más el trabajo académico y formativo es difundir la creatividad desde los mismos creadores. Para ello, es posible plantear espacios con curadoría estudiantil, conectando a Confibercom con iniciativas estudiantiles que pueden existir, o que están esperando un espacio para manifestarse. Espacios como estos no tienen que limitarse a difundir aquello que está siendo creado fuera de la academia, sino que también puede servir como enlace con los trabajos que con fines didácticos son realizados por los mismos docentes.

En ambos casos, el trabajo puede ser hecho con recursos básicos: existen universidades que cuentan con espacios digitales que pueden ser usados para actividades de este tipo, y si bien los recursos necesarios no son inmediatamente aparentes, es posible promover estas iniciativas, o versiones locales de las mismas, para buscar que logren sostenibilidad a partir de los esfuerzos concretos que grupos de la comunidad académica realicen, para luego buscar convertirlos en programas con financiamiento una vez ya encaminados.

La promoción del intercambio directo por parte de los docentes es relativamente más simple y al mismo tiempo requiere un esfuerzo más sofisticado. Herramientas como Mendeley permiten que el archivo personal de un suscriptor sea analizado y conectado a través de una base de datos colectiva a aquellos usuarios que tienen intereses similares, como funcionan también servicios de música por demanda tales como Spotify. El poder de Mendeley es que hace visible, conexiones que existen como potencia, en la coincidencia de temas y métodos de trabajo que dos 
académicos que comparten similares bibliografías en sus computadoras indicaría; a pesar de su compra por Elsevier, uno de los gigantes de la publicación académica, sigue siendo una herramienta valiosa. A través de herramientas como esta, es posible hacer explícitas las redes que deberíamos crear y que tradicionalmente son complejas y caras de montar.

A diferencia de las similitudes que pueden emerger de usar redes más formalizadas, como las que sistemas como Lattes/Scienti habilitan, o a través de sistemas nacionales de Ciencia y Tecnología que afilian a investigadores a grupos de trabajo, las herramientas de descubrimiento como Mendeley sirven para conectar de maneras no evidentes a académicos que pueden no formar parte de las redes ya establecidas pero que, a pesar de diferencias de estilos o aproximaciones, están trabajando temas similares, como la bibliografía indicaría. Obviamente no se trata de habilitar o promover una sola herramienta, sino de fomentar que estas experiencias de intercambio y conexión no formal se produzcan.

Confibercom puede ser un mecanismo para el descubrimiento y el intercambio, pero también tiene que ser una fuerza política para fomentar aquello que puede hacer más fácil el trabajo colectivo. La combinación de promoción activa, intelectual y práctica del acceso abierto, de la identificación de nuevos contenidos y de la utilización de nuevas herramientas de intercambio menos formal que aquellas que promueven las redes ya existentes puede ser lo que necesitamos para reforzar la integración intelectual de nuestra región, no limitándonos a la integración formal, entre instituciones, sino fomentando la manera más efectiva de conectarnos: entre individuos compartiendo proyectos, ilusiones y nuevas rutas de conocimiento colectivo.

\section{Referencias}

Berger, M. (2017). Everything you ever wanted to know about predatory publishing but were afraid to ask. In D. M. Mueller (Ed.), At the helm: Leading transformation: The proceedings of the ACRL 2017 conference (pp. 206-217). Association of College and Research Libraries. http://www.ala.org/acrl/sites/ala.org.acrl/files/content/conferences/confsandpreconfs/2017/EverythingYouEverWantedtoKnowAboutPredatoryPublishing.pdf

Borgman, C. (2007). Scholarship in the digital age. MIT.

Dutton, W. (2013). Internet studies: The foundations of a transformative field. In W. Dutton (Ed.), The Oxford handbook of Internet Studies. Oxford University Press. https://doi.org/10.1093/oxford$\mathrm{hb} / 9780199589074.013 .0001$

Fischman, G. ,\& Alperin, J. P. (2015). Sobre luces y sombras: Las revistas científicas hechas en Latinoamérica. In G. Fishman, J. P. Alperin, A. M. Cetto, J. O. Alonso-Gamboa, A. Packer, E. Aguado-López, \& N. da Silva Ávila (Eds.), Hecho en Latinoamérica: Acceso abierto, revistas académicas e innovaciones regionales (pp. 13-18). CLACSO.

Fuchs, C. (2014). Social media: A critical introduction. Sage. 
Meyer, E., \& Schroder, R. (2013). Digital transformations of scholarship and knowledge. In W. Dutton (Ed.), The Oxford handbook of internet studies. Oxford University Press. https://doi.org/10.1093/oxford$\mathrm{hb} / 9780199589074.013 .0001$

Safi, M. (2014, 25 de noviembre). Journal accepts bogus paper requesting removal from mailing list. The Guardian. https://www.theguardian.com/australia-news/2014/nov/25/journal-accepts-paper-requesting-removal-from-mailing-list 